\title{
Les conflits d'usage du cadre de vie dans les espaces ruraux et la décision publique locale
}

Éléments pour une analyse économique

Land-use conflicts about living environment in rural area and local public

choice. Elements for an economic analysis

\section{Philippe Jeanneaux et Philippe Perrier-Cornet}

\section{(2) OpenEdition}

\section{Journals}

Édition électronique

URL : http://journals.openedition.org/economierurale/458

DOI : 10.4000/economierurale.458

ISSN : 2105-2581

Éditeur

Société Française d'Économie Rurale (SFER)

Édition imprimée

Date de publication : 1 juillet 2008

Pagination : 39-54

ISSN : 0013-0559

\section{Référence électronique}

Philippe Jeanneaux et Philippe Perrier-Cornet, «Les conflits d'usage du cadre de vie dans les espaces

ruraux et la décision publique locale », Économie rurale [En ligne], 306 | Juillet-août 2008, mis en ligne le 01 juillet 2010, consulté le 10 décembre 2020. URL : http://journals.openedition.org/economierurale/ 458 ; DOI : https://doi.org/10.4000/economierurale.458 


\section{Les conflits d'usage du cadre de vie dans les espaces ruraux et la décision publique locale Éléments pour une analyse économique}

Philippe JEANNEAUX • ENITA Clermont-Ferrand, UMR Mutations des activités, des espaces et des formes d'organisation dans les territoires ruraux (Métafort)

Philippe PERRIER-CORNET • INRA Montpellier, UMR Marchés, organisation, institutions et stratégies d'acteurs (Moïsa)

\section{Contexte des conflits d'usage du cadre de vie résidentiel}

D epuis les années 1960, le développement résidentiel consécutif au processus d'étalement urbain modifie la physionomie des campagnes. Les campagnes françaises sont de plus en plus recherchées pour la qualité de leur cadre de vie en s'affirmant comme des lieux de résidence pour un nombre croissant de Français. Dans le même temps, la place de l'agriculture en termes d'emplois, de création de richesses et d'organisation des territoires ruraux reste importante, mais se réduit, alors que la protection de la nature conduit à reconnaître de plus en plus aux campagnes leur caractère d'espaces naturels (Perrier-Cornet, 2002).

Entre 1999 et 2004, le parc de logements s'est accru dans les espaces périurbains et ruraux à un rythme annuel $(1,1 \%)$ plus rapide que celui des pôles urbains $(+0,9 \%)$. Les migrations résidentielles des ménages vers les espaces périurbains et ruraux sont fortement motivées par la recherche d'une qualité de vie, que l'on explique généralement par la baisse des coûts de transport, l'augmentation du niveau de vie et la rente foncière plus faible ${ }^{1}$ (Plateau et Rakotomalaia, 2005). Les résidants recherchent un cadre de vie résidentiel dont l'originalité

1. D'autres éléments du contexte l'ont rendu plus clément pour expliquer les choix des ménages sur la période 1998-2002 : la baisse des taux d'intérêt, la croissance du pouvoir d'achat, le recul du chômage, la mise en place des prêts à taux zéro (PTZ) (Daubresse, 2003). est d'être agencé autour de trois composantes clés qui s'emboîtent et qui composent le capital spatial habitat (Lévy, 1994) :

- le logement (la maison individuelle en propriété et son espace vert attenant) ;

- l'accès au centre d'emploi permis par la voiture ;

- la nature préservée composée d'aménités (les paysages, le calme, le silence, le bienêtre, l'air pur) pouvant être affectée par des nuisances.

Les territoires ruraux connaissent de ce fait une concurrence croissante pour leurs usages et leur contrôle, qui peut aller jusqu'à déboucher sur des oppositions ouvertes, communément qualifiées de conflits d'usage ou de conflits environnementaux (Charlier, 1999). Les oppositions sont très variables : ici une association de riverains s'oppose au projet de création d'un site de stockage de déchets ménagers, là un propriétaire foncier conteste le passage de randonneurs sur ses terres, ailleurs encore un maire sous la pression de ses administrés refuse de délivrer un permis de construire nécessaire à l'extension d'une porcherie. Pour de nombreux usagers résidentiels, opposés à tous projets et toutes activités susceptibles de détériorer leur cadre de vie, la campagne ne doit être qu'un havre de paix : "À quoi bon vivre ou s'installer à la campagne, si c'est pour voir détruire ce à quoi on tient ou ce qu'on y vient chercher?»(Hussenet, 2002) ${ }^{2}$.

2. $C f$. page 4 . 
Ces situations polémogènes ont la particularité et le point commun d'avoir un lien avec les ressources environnementales localisées d'un espace composant le cadre de vie des habitants. Elles ne relèvent pas que de situations de dispute entre voisins ; elles ont aussi une dimension collective et publique. Dans nombre de cas, il apparaît en effet que les usagers du cadre de vie résidentiel contestent des décisions publiques qu'ils rendent responsables de leur situation : en réglementant, en interdisant ou autorisant certains usages, les choix des élus et/ou de leur administration ne peuvent satisfaire unanimement les administrés. L'intervention publique locale au nom de l'intérêt général crée inéluctablement des perdants. Se considérant alors comme les victimes d'une injustice, les perdants contestent la distribution inégale des aménagements et des projets mis en œuvre ou autorisés par les décideurs publics locaux (Richman et Boerner, 2004).

La notion de conflit d'usage développée dans cet article contient quelques spécificités à préciser. Tout d'abord, à l'instar de Simmel (1995), nous traitons le conflit comme un élément inhérent aux sociétés, tout comme le compromis ou l'entente. Le conflit n'est pas une anomalie de la vie des sociétés, il en fait partie intégrante. Le conflit est un temps (possible) de rééquilibrage et de structuration des forces d'adversaires rationnels (Deutsch, 1994). Il est utile (mais pas indispensable) dans certaines situations, parce qu'il est le principal vecteur d'ajustement des règles et des institutions de toute société.

Ensuite, les conflits que nous traitons ne se limitent pas aux conflits d'usage dans leur version économique étroite de surexploitation des ressources (Libecap, 1989 ; Point, 1997). La notion de conflit d'usage que nous retenons englobe plus largement l'ensemble des problèmes de nuisances. Ce terme générique recouvre pour nous l'idée de confrontation entre usagers lors de la poursuite du même but de manière compé- titive ou lors de la poursuite de buts antagonistes, pour l'usage d'un bien environnemental localisé, que la compatibilité entre les usages soit réelle ou potentielle. C'est avec cette même acception qu'un groupe de travail du Commissariat général du plan (Guérin, 2005) a analysé les situations conflictuelles avec pour objectif d'évaluer leurs implications pour la décision publique.

Dans ce contexte, notre recherche est donc centrée sur les conflits d'usage du cadre vie résidentiel dans les espaces ruraux et périurbains, que nous définissons comme des situations polémiques dans lesquelles les metteurs en cause et/ou les mis en cause sont des usagers résidentiels. L'objet de la recherche ne concerne pas ici l'évaluation économique des dommages liés à la dégradation du cadre de vie résidentiel. Elle vise principalement à éclairer les effets des conflits d'usage sur l'allocation de ressources communes locales à dimension environnementale par le décideur public local. En regard du cadre d'analyse et de la méthodologie adoptée que nous développons dans une première partie, nous nous focalisons donc plus précisément sur les conflits pour lesquels les autorités publiques en charge des affaires locales sont concernées. Notre réflexion s'appuie sur une étude empirique menée sur le département du Puy-de-Dôme à partir de deux sources originales, dont les principales observations sont rassemblées dans les trois parties suivantes de cet article.

\section{Cadre d'analyse et méthodologie}

\section{Les dimensions matérielle et institutionnelle des conflits}

Si nous nous intéressons plus particulièrement aux conflits impliquant les autorités publiques locales, c'est parce que le décideur public apparaît comme un mode possible d'allocation de ressources communes locales à dimension environnementale. Celles-ci sont constitutives du cadre de vie résidentiel. Les situations polémogènes qui visent à 
remettre en cause des choix publics locaux concernant ces ressources vont aller de pair avec la montée en puissance des usages résidentiels et la transformation de la physionomie des espaces périurbains et ruraux. Ceci étant, nous sommes bien conscients que tous les conflits ne mettent pas en scène le décideur public. Nous verrons plus loin qu'une des sources que nous avons mobilisées, les articles publiés par la presse quotidienne régionale $(\mathrm{PQR})$, permet de situer la catégorie des contestations des autorités publiques locales par rapport à l'ensemble de la conflictualité sur un territoire donné.

La problématique que nous développons suggère que les conflits d'usage mettent en jeu deux dimensions, matérielle et institutionnelle, qui peuvent se présenter de façon isolée ou combinée (Jeanneaux, 2006).

- Tout d'abord, la première dimension, la plus générique, que nous qualifions de matérielle pose que les conflits d'usage relèvent principalement de problèmes d'effets dommageables matériels (pollution, limitation d'accès, encombrement...) produits par certains agents qui exercent une activité, en tirent des avantages, en faisant supporter (non intentionnellement) à d'autres des désagréments ou des coûts non compensés (Baumol et Oates, 1988), auxquels ces derniers n'ont pas consenti. Dans notre analyse, le développement des usages résidentiels remet en cause l'appropriation par certains usages des attributs des ressources environnementales localisées. Si les effets des actions des autres se traduisent par des effets matériels dommageables réels ou potentiels, cela signifie qu'un nouvel usage apparaît ou qu'un usage existant est modifié. Ces évolutions se traduisent par des incompatibilités d'usage et par une mise à l'agenda de valeurs d'usage des ressources jusque-là ignorées. Cette délimitation nous permet de proposer que les conflits d'usage relèvent de la catégorie générique des effets externes technologiques négatifs (Catin, 1985 ; Mishan, 1969). Intuitivement, nous abordons le conflit comme le signal du refus des agents d'être les victimes d'un effet dommageable réel ou potentiel. Dès que le niveau de nuisance atteint est jugé socialement inacceptable, il devient un problème environnemental demandant une correction (Ruff, 1970).

- La deuxième dimension, plus spécifique à notre recherche, se focalise sur l'origine institutionnelle, publique, des conflits d'usage. L'action des pouvoirs publics pour répondre à la demande des citoyens concernant leur cadre de vie se décline selon deux grandes modalités :

- la fourniture de biens publics locaux ;

- la fourniture de règles d'usage permettant d'exploiter les fonctionnalités des ressources environnementales localisées.

Dans les deux cas, les autorités publiques locales doivent mettre en œuvre, via la réglementation administrative, les très nombreuses règles d'usage contraignantes pour organiser la coexistence des usages dans leur diversité. Les politiques publiques en matière d'urbanisme, d'environnement ou d'agriculture s'appuient très largement sur des instruments qui limitent les actions des agents économiques comme :

- le régime des installations classées pour la protection de l'environnement (ICPE) ; - les zonages (POS, PLU, PPRN, ZNIEFF, Natura 2000...) ;

- les permis de construire... ;

- les schémas directeurs (assainissement, carrière, déchets...) ;

- les procédures d'expropriation lors de déclaration d'utilité publique de projets d'aménagement.

Cette dimension institutionnelle nous conduit à développer l'analyse économique de la décision publique. Elle concerne la fourniture par la puissance publique locale d'instruments administratifs de réglementation des usages de biens environnementaux localisés, et de certains biens publics locaux, dans un régime de démocratie indirecte. Dans un tel régime, eu égard à la taille importante du groupe, les citoyens élisent 
des représentants chargés d'administrer la cité conformément aux intérêts des mandants. Le décideur public en modifiant la structure des droits provoque des changements sur la distribution de la richesse et du pouvoir politique qui ne peuvent respecter l'unanimité parétienne (Jessua, 1991), même si ces décisions sont fondées sur la recherche de l'intérêt général. Le décideur public transmet un effet externe politique négatif (Buchanan et Tullock, 1962). L'élection n'est plus suffisante pour révéler aux mandataires les préférences des électeurs, et l'action en lobbying informationnel qui s'enclenche avec le conflit, corrige la défaillance du dispositif électoral (Vigouroux, 1999). L'action collective des groupes de pression s'assimilera ici à une forme de marchandage politique permettant aux usagers (électeurs) d'exprimer par la contestation leurs préférences afin que le personnel politique ou l'administration en tiennent compte pour éventuellement ajuster la décision et modifier la répartition des pertes entre agents. Le conflit d'usage apparaît ainsi comme un temps utile au décideur public pour affiner sa connaissance des préférences des administrés. L'expression de la contestation des choix publics locaux utilise deux leviers principaux de pression en plus du signalement des préférences des citoyens par le vote :

- le premier concerne l'action médiatique qui s'exerce par les manifestations de rue, le pétitionnement, ou encore les réunions publiques. L'objectif principal de ce lobbying informationnel consiste à élargir l'espace des citoyens directement concernés et qui refusent d'être les victimes d'un préjudice matériel et les perdants de la décision publique ;

- le second levier concerne la contestation qui s'exerce lors des recours en contentieux devant les juridictions administratives et qui vise à faire annuler les décisions administratives des autorités publiques locales.

Les deux dimensions matérielle et institutionnelle peuvent ou non se combiner en se déclinant selon une diversité de situations, du simple conflit de voisinage interindividuel ne mettant pas en scène le décideur public, au conflit dans lequel celui-ci est à l'origine simultanément de l'effet externe technologique négatif et de l'effet externe politique négatif. Après les avoir situées dans l'ensemble des conflits, nous nous focaliserons sur les situations qui combinent les deux dimensions, matérielle et institutionnelle, dans lesquelles sont mises en scène les autorités publiques.

\section{Deux sources originales pour matérialiser les conflits à l'échelle d'un département}

Pour discuter nos propositions en matière de contestation des choix publics locaux ayant un impact sur la qualité du cadre de vie résidentiel, nous avons dû organiser un recueil des conflits à partir de deux sources originales et complémentaires, afin de rassembler un matériau empirique exploitable : - la première vise à cerner l'action médiatique, par l'analyse de la presse quotidienne régionale $(\mathrm{PQR})$ : nous avons dépouillé le quotidien La Montagne sur le département du Puy-deDôme sur une période de quatre ans ;

- la seconde concerne le recours en contentieux contre la décision publique : elle a été appréhendée par les jugements du tribunal administratif (dépouillés sur la même période dans le Puy-de-Dôme).

Les exploitations quantitatives de la presse ou des tribunaux sont rares et assez différentes de notre approche. Charlier (op. cit.) a produit une analyse de géographie sociale originale de la conflictualité environnementale pour la France à partir de la revue spécialisée Combat Nature. Pour notre part, nous avions réalisé une première exploration de la conflictualité (Guillain, 2001) à partir de trois quotidiens régionaux. Par la suite, Torre et al. (2006) ont repris cette approche et développé les analyses des conflits et leurs modes de résolution sur une dizaine de départements français à partir de la presse quotidienne régionale ou de traitements des arrêts du Conseil d'État et de 
la Cour de cassation. Cependant, il apparaît que la majorité des travaux sur les conflits ont d'abord développé des approches monographiques de conflits emblématiques (Caron et Rialland, 2001).

Les deux sources mobilisées présentent l'avantage d'identifier les caractéristiques d'émission et de réception des effets externes technologiques et politiques à l'origine des conflits, mais aussi d'exposer les stratégies de marchandages des droits d'usage contenues dans la réglementation administrative. La PQR présente l'avantage d'exposer la très grande diversité des conflits d'usage qui sont médiatisés, des troubles anormaux de voisinage aux contestations des grandes infrastructures publiques. Elle permet ainsi de situer, dans le champ des affaires médiatisées, l'importance des contestations des autorités publiques locales par rapport à l'ensemble des conflits d'usage. Elle donne une vue d'ensemble de la conflictualité mais reste limitée aux affaires médiatisées. En regard de notre objectif qui se centre sur les conflits mettant en jeu la décision publique, nous avons exploré les jugements d'un tribunal administratif (TA) portant explicitement sur ce champ. Ils ont l'avantage d'être une source extrêmement précise de la contestation des décisions publiques, y compris ceux qui ne sont pas médiatisés.

Par ailleurs, la PQR donne la possibilité de mener une analyse longitudinale des conflits permettant de mesurer l'issue effective du conflit, ce que ne permet pas le traitement des conflits repérés au TA. En effet, si les jugements du TA informent sur la validité des décisions des élus, ils ne permettent pas d'apprécier les effets réels du jugement sur les usages des ressources.

Ces deux sources permettent de décrire, dater et localiser les conflits d'usage du cadre de vie dans un territoire donné. Notre terrain d'étude, l'ensemble du département du Puy-de-Dôme, a été choisi parce que, d'une part, l'accès aux sources nous a été facilité et que, d'autre part, une partie non négligeable de ce département est marquée par un fort mouvement d'étalement urbain et résidentiel entraînant une forte pression humaine pour l'usage des ressources localisées environnementales.

\section{La méthodologie du recueil des données}

La recension des conflits dans la PQR a été effectuée à partir du fonds d'archives de la bibliothèque municipale inter-universitaire de Clermont-Ferrand pour une période de quatre ans (janvier 1999 à décembre 2002). L'essentiel de l'information sur les conflits d'usage a été repéré dans les pages d'actualités départementale et locale. Le recueil des articles de presse a porté sur les conflits mettant en jeu l'usage de ressources localisées sur les espaces ruraux, périurbains et urbains. Pour être retenu dans notre base comme conflit, la situation présentée dans le quotidien, outre sa localisation dans le département, devait répondre à quatre critères : - la citation explicite d'un problème matériel effectif ou potentiel d'usage de biens environnementaux (une nuisance, une exclusion d'un site, une interdiction d'accès, un différend pour l'usage d'une ressource...) ; - des parties en opposition (un contestataire, un contesté) ;

- des usages identifiés pour chaque partie ; - un élément de signalement du conflit (pétition, manifestation de rue, réunion, dépôt de plainte...)

Un premier recensement exhaustif sur le département du Puy-de-Dôme a permis de recenser 430 articles de presse correspondant à 148 conflits d'usage (un même conflit peut être suivi dans le temps et faire l'objet de plusieurs articles) tous types d'espaces confondus. Les quatre cinquièmes d'entre eux (118 conflits) sont situés dans les espaces ruraux ou périurbains : les conflits d'usage médiatisés par ce canal concernent essentiellement les campagnes. Nous avons ensuite exclu de l'analyse les situations conflictuelles médiatisées qui, dans les campagnes, ne mettent 
pas explicitement en scène un usager (ou groupe d'usagers) du cadre de vie résidentiel (51 conflits : entre agriculteurs, entre agriculteurs et industriels, entre chasseurs et agriculteurs...). Nous avons en définitive travaillé sur 67 des 118 conflits recensés, soit près de $60 \%$ des conflits médiatisés dans le rural et le périurbain : la majorité des situations polémogènes médiatisées aujourd'hui dans le rural concerne bien le cadre de vie résidentiel.

Les conflits d'usage du Puy-de-Dôme recueillis au tribunal administratif de Clermont-Ferrand ont été préalablement recensés à partir de six postes de la nomenclature des juridictions administratives : agriculture, environnement, expropriation, domaine-voirie, police et urbanisme. Ce premier filtre a permis de constituer une première base de 1000 jugements. Pour être retenue dans notre base comme conflit, la situation présentée dans les jugements du tribunal administratif devait répondre aux trois premiers critères présentés ci-dessus.

La collecte au tribunal administratif a permis de retenir le verbatim de 554 jugements correspondant à 416 conflits d'usage pour une période de cinq années (janvier 1998 à décembre 2002). Là également, les quatre cinquièmes sont localisés dans des espaces ruraux ou périurbains $(\mathrm{N}=336)$. Parmi ceux-ci, un peu plus de la moitié $(\mathrm{N}=183)$ relèvent spécifiquement de conflits d'usage du cadre de vie résidentiel (n'ont, en particulier, pas été pris en compte les nombreux recours d'agriculteurs contre un remembrement, quand il n'y avait pas d'enjeu résidentiel dans le litige).

Ces deux sources intéressantes, mais excessivement lourdes à exploiter, présentent quelques limites qui peuvent nuancer les enseignements que nous avons tirés. L'exploitation du quotidien La Montagne a permis de constituer une base très variée de la conflictualité. Nous avons pu identifier toutes les étapes des conflits, de leur survenance à leur issue. Néanmoins, la production des nouvelles est par construction hétéro- gène, non exhaustive et parfois partiale. De plus, la PQR ne s'intéresse qu'aux conflits que les protagonistes veulent médiatiser. La rédaction du quotidien contrôle et sélectionne l'information. Les nouvelles que nous traitons correspondent donc à une information de seconde main, contenant parfois des interprétations discutables par les protagonistes des conflits. Le tribunal administratif s'est révélé être aussi une source extrêmement riche. Le contenu en information des décisions de justice est certes juridique, mais il comporte aussi des éléments non juridiques. Il permet d'extraire des informations concernant les agents, leurs préférences, les moyens de droit et la décision du juge, l'intérêt à agir, les règles d'usage en jeu, la nature des compensations.

\section{Une grande diversité de conflits d'usage du cadre de vie résidentiel}

\section{Les conflits médiatisés dans les campagnes}

Les 67 conflits médiatisés d'usage du cadre de vie résidentiel dans les espaces périurbains et ruraux représentent donc près de 6 conflits sur 10. Ils sont les signaux des mutations de campagnes de plus en plus attractives par le capital spatial habitat qu'elles offrent alors que dans le même temps elles restent le siège d'activités productives encore nombreuses. Ils correspondent à des situations dans lesquelles les résidants s'opposent principalement aux activités productives et à leurs effets externes négatifs réels ou supposés. Parmi les exemples les plus significatifs rencontrés, l'exploitation de carrières est souvent contestée, essentiellement pour les dangers et le bruit des camions transportant les matériaux extraits. L'exploitation de sites de stockage de déchets ou d'autres produits perçus comme dangereux (farines animales, explosifs, engrais, combustibles) est refusée dans plusieurs sites au nom de la préservation de la santé ou des 
ressources vitales comme l'eau. Les élevages hors sols (porcheries et ateliers avicoles) sont rejetés pour leurs nuisances olfactives...

Les motifs qui conduisent les récepteurs des externalités à se déclarer victimes relèvent, dans la presse, essentiellement de problèmes de pollution (tableau 1). Ce sont principalement des entreprises, des «producteurs » qui en sont rendus responsables : la montée en puissance des usages résidentiels se heurte à la présence d'autres usages notamment productifs. Dans les articles de La Montagne, les principaux mis en cause sont les entreprises (38 cas sur 67), puis les collectivités locales (commune ou syndicat intercommunaux).

Les récepteurs des effets externes technologiques, c'est-à-dire ceux qui déclarent être des victimes, ou qui revendiquent un droit d'usage symétrique (au sens coasien) entravé par un autre usager émetteur, ont des statuts très divers. Les opposants sont d'abord des associations circonstancielles locales (1 cas sur 2), mais également des collectivités locales (1/4 des cas) qui, sous la pression, se font les relais des préoccupations de leurs administrés pour le maintien du cadre de vie résidentiel. La presse quotidienne ne fait que peu écho aux conflits interindividuels et privilégie les actions collectives de contestation.

La prise de parole lors du conflit permet à une catégorie d'agents de signaler la réception d'un dommage, autrement dit de signaler qu'ils sont eux-mêmes des usagers de certains attributs d'un bien environnemental localisé, et qu'ils aspirent à faire reconnaître des droits d'usage sur cette ressource. La prise de parole est donc un vecteur des préférences d'une catégorie d'agents. Les conflits portés par les usagers résidentiels remettent en cause les usages préférés et obligent à obtenir le réexamen des normes administratives et l'exercice de nouveaux arbitrages sur les droits d'usage particulièrement contrôlés par les autorités publiques locales.

Certains usages sont en effet négligés ou éprouvent des difficultés à être acceptés, tant ils sont confrontés à des usages locaux exploitant les ressources nécessaires au fonctionnement de la cité (activités d'extraction de matériaux, de traitement des déchets, de stockage de l'énergie, d'infrastructure de transport et d'activités industrielles). Les entreprises, comme les collectivités locales, paraissent contrôler les ressources et ignorer les autres usagers pour des raisons spécifiques. Tout d'abord, les firmes, par leurs activités économiques, sont identifiées comme productrices de richesses et d'emplois. Cette position leur donne un pouvoir sur l'usage des biens environnementaux localisés qui pourrait expliquer la difficulté pour les autres usages, sans intérêts économiques apparents, à être reconnus comme des usages possibles. Ensuite, les collectivités territoriales, investies d'une mission de service public local, peuvent négliger les intérêts des résidants au nom de l'intérêt général et engendrer la contestation.

Tableau 1. Dans la presse quotidienne régionale, motif principal conduisant à se déclarer victime

\begin{tabular}{l|c|c}
\hline Motif conduisant à se déclarer victime & Nb. cit. & Fréq. \\
\hline Problème de dégradation des ressources naturelles (eaux, sols...) & 31 & $46 \%$ \\
\hline Problème de bruit & 15 & $22 \%$ \\
\hline Problème d'odeur & 10 & $15 \%$ \\
\hline Problème d'utilisation ou d'occupation d'un bien & 5 & $8 \%$ \\
\hline Problème esthétique et de paysage & 4 & $6 \%$ \\
\hline Problème de voisinage & 2 & $3 \%$ \\
\hline TOTAL OBS. & 67 & $100 \%$ \\
\hline
\end{tabular}




\section{Les conflits d'usage dans le contentieux administratif}

Tous espaces confondus, avec près de 6 cas sur 10 (239 conflits sur 416), les conflits d'usage du cadre de vie résidentiel sont également les plus nombreux au tribunal administratif. Nous retrouvons une proportion comparable pour les conflits d'usage localisés dans les seuls espaces périurbains et ruraux (183 sur 336). À l'instar de nos observations dans la PQR, cette part importante de conflits signale là aussi l'intérêt que les résidants de ces espaces attribuent à leur cadre de vie résidentiel.

Pour ces 183 conflits, la fraction la plus importante du contentieux administratif ( 2 cas sur 3) concerne la contestation de zonage (plan d'occupation des sols ou de remembrements posant un problème de constructibilité) dont le point commun serait d'être des opérations accusées d'entraver des projets futurs de construction de maisons individuelles (tableau 2). D'autres requérants contestent, par ailleurs, la mise en place par les autorités locales de servitudes ou d'expropriations réalisées au nom de l'intérêt général pour construire des voies de circulation, ou pour permettre l'installation de réseaux divers (gaz, eau, électricité, téléphone).

On rencontre également des résidants qui s'opposent aux projets de construction de maison d'un futur voisin en revendiquant l'équivalent de servitudes d'air, de lumière et de paysage. Aussi, les motifs qui condui- sent les récepteurs des externalités à se déclarer victimes relèvent principalement, devant le tribunal administratif, de problèmes de restriction d'usage d'un bien privé par les décideurs publics

$\mathrm{Si}$ du fait de sa fonction principale, le contentieux administratif compte une majorité de conflits dont le responsable du préjudice est une autorité publique locale, il ressort que les requêtes du tribunal administratif sont, pour l'essentiel, portées par des individus isolés non organisés (7 cas sur 10) alors que les associations généralistes ou dédiées sont peu présentes.

Les deux sources donnent ainsi une vision quelque peu différente de la conflictualité dans les campagnes. Certes, on retrouve dans les jugements du TA une part des conflits médiatisés dans la PQR (ceux dans lesquels les pouvoirs publics sont d'une façon ou d'une autre mis en cause), mais on y découvre aussi une autre forme de conflictualité, portée individuellement par des résidents en désaccord avec des réglementations et des choix publics locaux.

\section{Deux grandes catégories de contestation des décideurs publics locaux}

Pour éclairer la contestation des choix publics locaux, nous nous sommes appuyés sur la combinaison des grilles d'analyse des externalités politiques et technologiques négatives.

Tableau 2. Devant le tribunal administratif, motif principal conduisant à se déclarer victime

\begin{tabular}{l|c|c}
\hline Motif conduisant à se déclarer victime au TA & Nb. cit. & Fréq. \\
\hline Problème de restriction d'usage d'un bien privé (refus de permis de construire) & 86 & $47 \%$ \\
\hline Problème de dégradation des ressources naturelles (eaux, sols...) & 33 & $18 \%$ \\
\hline Problème d'utilisation ou d'occupation d'un bien (servitudes...) & 30 & $16 \%$ \\
\hline Problème esthétique et de paysage & 15 & $8 \%$ \\
\hline Problème de voisinage & 14 & $8 \%$ \\
\hline Problème d'odeur & 4 & $1 \%$ \\
\hline Problème de bruit & 1 & $1 \%$ \\
\hline TOTAL OBS. & 183 & $100 \%$ \\
\hline
\end{tabular}

Source : dépouillement par les auteurs des jugements du TA du Puy-de-Dôme (période 1998-2002) 
Parmi les 67 situations conflictuelles repérées dans la presse quotidienne régionale (PQR), pour lesquelles au moins un des antagonistes est un usager du cadre de vie résidentiel, l'analyse empirique montre que 32 conflits relèvent de situations présentant uniquement des effets dommageables matériels. Nous ne détaillons pas ici ces conflits qui ne mettent pas en scène les autorités publiques. Précisons toutefois que pour ces cas, les autorités publiques ne sont considérées ni comme victimes ni comme responsables des dommages, mais nous avons observé qu'elles sont généralement sollicitées dans les conflits pour assurer la médiation entre les antagonistes.

Nous avons concentré notre analyse sur les 35 autres situations pour lesquelles les décideurs publics locaux ou centraux sont mis en cause d'une façon ou d'une autre par des «perdants » locaux. Ce cas de figure correspond aussi aux 183 conflits que nous avons recensés au tribunal administratif (dans lesquels on retrouve d'ailleurs une bonne partie des 35 conflits médiatisés dans la PQR, qui ont fait l'objet d'un recours en contentieux administratif).

Deux grandes catégories de contestation des décideurs publics locaux apparaissent : - soit ils sont indirectement mis en cause, car en prenant des décisions en faveur d'agents privés, ils transmettent des effets externes politiques négatifs en créant des perdants non compensés. C'est un agent privé dont l'usage est permis par une autorité publique qui est à l'origine de la création d'une victime en lui transmettant un effet externe technologique négatif ;

- soit ils sont directement mis en cause quand ils fournissent directement un bien public local et créent simultanément des victimes et des perdants non compensés.

\section{Première catégorie}

Les autorités publiques locales, émettrices d'un effet externe politique négatif, mais pas de l'effet externe technologique

Dans ces cas (16 sur 35 observés dans le journal La Montagne et 53 sur 183 cas pour le tribunal administratif, $c f$. figure 1), une firme ou un particulier transmet un effet externe technologique négatif et crée une victime parce qu'une autorité publique autorise son activité, jugée indésirable par le récepteur. La puissance publique locale transmet alors un effet externe politique négatif, parce qu'elle autorise un usage qui a la particularité d'être d'ordre privé (même si l'intention du régulateur est de préserver la population des nuisances). Les autorités publiques créent des perdants qui considèrent que des droits à polluer ont été fournis et risquent d'être à l'origine d'une possible détérioration de leur cadre de vie. Le cas des autorisations d'installations

Figure 1. L'autorité publique locale, émettrice d'un effet externe politique négatif mais pas de l'effet externe technologique

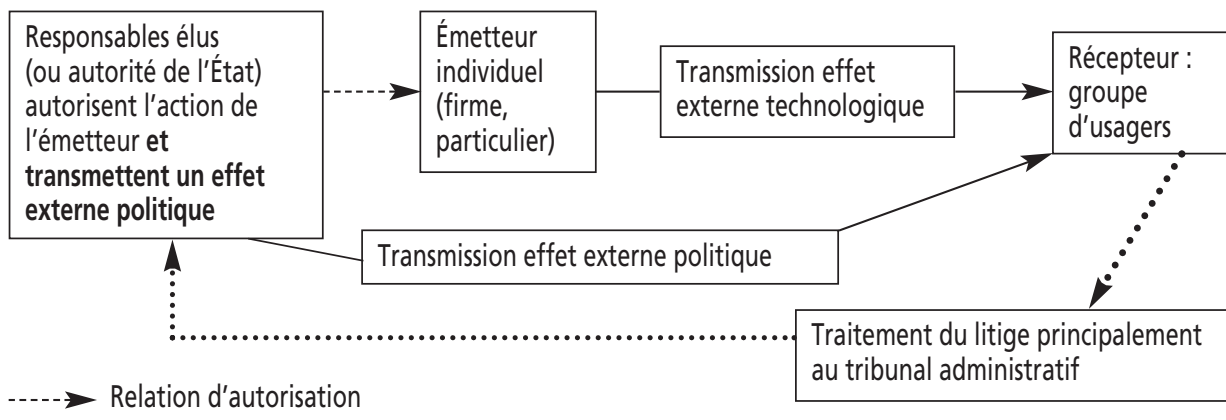

$\longrightarrow$ Sens de l'externalité 
classées pour la protection de l'environnement (ICPE) est emblématique de cette situation. Bien entendu, l'acte administratif n'est pas seul en cause dans l'histoire, car c'est tout d'abord le projet d'un agent privé, l'émetteur individuel, qui déclenche l'action de la puissance publique. Mais force est de constater que l'action de contestation se concentre en priorité sur la décision de l'autorité publique.

Prenons le cas d'une petite commune rurale où une association de riverains a été créée pour empêcher l'implantation d'une carrière. Les riverains redoutent les nuisances sonores et olfactives des camions et refusent la dégradation de leur cadre de vie. Ils sont supposés recevoir un effet externe technologique négatif émis par l'exploitant carrier. De plus, l'autorisation d'exploiter, en passe d'être délivrée par le préfet, s'assimile à la réception d'un effet externe politique négatif. Le préfet crée des perdants en délivrant des droits d'extraction de matériaux à la firme, et en négligeant les droits à un cadre de vie sans nuisances sonores engendrées par le trafic de camions dans la commune (50 poids lourds par jour). Les opposants ont manifesté, lors de l'enquête publique, leurs craintes en défendant un usage domestique des voies communales et contestent la décision du préfet au tribunal administratif.

\section{Deuxième catégorie}

Les autorités publiques locales, émettrices d'effets externes technologiques et politiques négatifs simultanés

Dans cette catégorie, les autorités publiques locales sont directement mises en cause quand elles fournissent directement un bien public local et créent simultanément des victimes et des perdants non compensés.

Nous avons observé 19 conflits pour cette catégorie dans la presse quotidienne (sur les 35 où la puissance publique est mise en cause) et 130 sur 183 au tribunal administratif (cf. figure 2). Tout d'abord, parce qu'ils ne connaissent qu'imparfaitement les préférences des administrés, les pouvoirs publics transmettent inéluctablement un effet externe politique négatif quand ils financent un bien public (une route, une usine d'incinération des déchets...), ou son fonctionnement, ou quand ils édictent et mettent en œuvre des règles d'usage locales (POS, PLU). Ensuite, simultanément, les pouvoirs publics transmettent une nuisance correspondant à une catégorie d'effet externe technologique négatif. Le responsable de l'émission de l'effet externe technologique et de l'effet externe politique est donc la même entité. Mais contrairement à la catégorie précédente, l'usage promu par le décideur local a la particularité d'être d'ordre public. Il s'agit d'une situation dite de rupture d'égalité des citoyens devant une charge publique.

Figure 2. L'autorité publique locale, émettrice d'effets externes technologiques et politiques négatifs simultanés

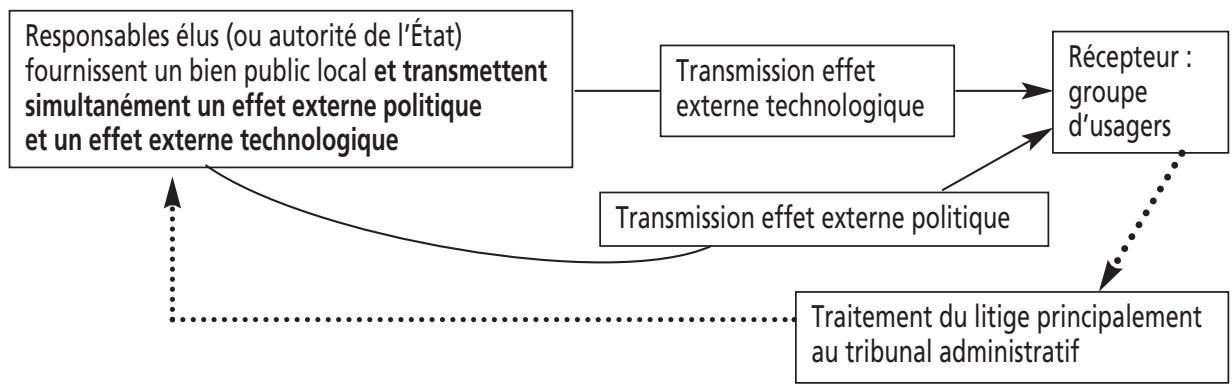

$\longrightarrow$ Sens de l'externalité

...... Sens du recours en contentieux

Source : conception des auteurs

48 • Économie RuRale 306/JuILLet-AOÛt 2008 
Par exemple, pour fournir certains biens publics locaux comme des infrastructures routières (pont sur la rivière Allier), les autorités publiques doivent obtenir le droit de construire la route. En plus de la difficulté d'exproprier et de dédommager les propriétaires fonciers, le décideur public doit obtenir le droit d'implanter une infrastructure produisant des nuisances. Un tel projet est contesté par des opposants, victimes du bruit du trafic, qui sont également les perdants du choix public (ici l'itinéraire de contournement).

\section{La stratégie des opposants Éviter d'être les perdants des choix publics locaux}

\section{Le problème de l'évaluation des dommages : vers l'absence de compensation des perdants}

L'incertitude sur les droits d'usage à l'origine de la création de victimes résulte d'un problème matériel lié aux caractéristiques des biens environnementaux. Ces biens sont complexes parce qu'ils sont dotés de multiples attributs sans droit de propriété toujours bien spécifié. Ils permettent des usages variés parfois difficilement contrôlables. La fréquence des usages, leur nature, leurs impacts sur les ressources (en termes d'irréversibilité, par exemple) (Passet, 1979), ou encore la diversité de leur expression spatiale (Jackman, 1975), pourront renforcer l'appropriation de la ressource par une catégorie d'usagers et provoquer un dommage subi par une autre catégorie d'usagers. Parce que les dommages sont difficilement évaluables, et les relations de causalité entre le dommage et l'action dommageable parfois ténues, l'émetteur pourra créer des victimes sans s'en rendre compte.

Par ailleurs, le caractère potentiel du dommage ne doit pas signifier que certains attributs n'ont pas de valeur d'usage, mais qu'ils peuvent correspondre à d'autres valeurs (valeur d'existence, de legs, d'option...). Les dommages hypothétiques correspon- dent à la catégorie des pollutions dites graduelles : la manifestation de l'atteinte au cadre de vie n'est pas concomitante à l'événement qui en est à l'origine. La dégradation du cadre de vie se réalise progressivement et est difficilement mesurable. Les caractéristiques des dommages compromettent l'exercice de la responsabilité. Cette situation renforce la minimisation de l'existence de victimes.

Nous observons que le type de préjudice subi a un effet sur la possibilité d'appliquer une règle de protection des droits et l'obtention de réparation des dommages. La règle de responsabilité s'applique préférentiellement aux situations accidentelles, concernant une population importante, et pour lesquelles l'occurrence est calculable par les assureurs. Or dans la quasi-totalité des situations observées dans la presse (33 cas sur 35), comme au tribunal administratif (182 cas sur 183), les riverains d'installations et d'activités à l'origine d'émissions continues ou récurrentes de polluants reçoivent plutôt des dommages chroniques. Les victimes sont par ailleurs restreintes à un groupe localisé dont l'étendue est trop limitée pour diviser les risques. Cette situation est à l'évidence incompatible avec une prise en charge possible par le système de l'assurance. En effet, la dégradation du cadre de vie est souvent perçue comme régulière, fréquente ou récurrente, au point de devenir habituelle. Ces dommages sont négligés et ne donneront pas accès à une compensation des victimes. En témoigne, en France, l'absence de couverture de nombreux dommages environnementaux par le secteur de l'assurance (Quirion, 1999). Par conséquent, devant cette absence inéluctable de compensation, les victimes potentielles vont agir pour éviter de devenir les perdants des choix publics locaux

Les choix publics (fourniture de biens publics, mise en application de la réglementation administrative) dans un monde avec grands groupes produisent nécessairement des gagnants et des perdants. Il ne 
Tableau 3. Attentes exprimées par les opposants (Presse régional quotidienne)

\begin{tabular}{l|c|c}
\hline Attentes des opposants PQR & Nb. cit. & Fréq. \\
\hline Abandon de l'usage ou du projet d'usage des mis en cause & 22 & $63 \%$ \\
\hline Aménagement de l'usage ou du projet d'usage des mis en cause & 10 & $28 \%$ \\
\hline Informations des mis en cause pour les metteurs en cause & 2 & $6 \%$ \\
\hline Compensation financière & 1 & $3 \%$ \\
\hline TOTAL OBS. & 35 & $100 \%$ \\
\hline
\end{tabular}

Source : dépouillement par les auteurs du quotidien La Montagne (période 1999-2002)

peut plus subsister de situations unanimement préférées. Les conflits analysés mettent en exergue de nombreuses situations de perdants non compensés cherchant à améliorer leur bien-être. Le comportement des perdants ne correspond toutefois pas à une recherche de dédommagement monétaire par les gagnants de la collectivité. En fait, il apparaît que les perdants potentiels devant la difficulté à être compensés et devant les difficultés à fuir les dommages eu égard à l'attachement au cadre de vie résidentiel, prennent la parole (Hirschman, 1970) pour infléchir ex ante les choix publics locaux afin de conserver leur bien-être.

Dans la PQR, nous avons relevé que les attentes des opposants visent essentiellement la modification de l'usage préjudiciable en cherchant son abandon (deux-tiers des cas) ou son aménagement (près de 3 cas sur 10) ( $c f$. tableau 3). Les aménagements attendus ne correspondent pas à une demande de compensation environnementale (par exemple, la mise en réserve d'un espace naturel contre un aménagement routier). Seule une situation conflictuelle concerne une demande de résolution par la voie de la compensation monétaire.
Du côté du tribunal administratif, nous n'avons repéré que 4 recours en plein contentieux permettant d'accéder à un dédommagement pécuniaire ( $c f$. tableau 4 ). En revanche, les attentes des contestataires relèvent du contentieux pour abus de pouvoir. Il s'agit de procès fait à un acte administratif dont l'objectif vise principalement à obtenir l'abandon de l'activité ou du projet contesté à l'origine des préjudices.

La demande d'abandon de projets d'usages de manière préventive ou de manière curative pour faire interdire une activité existante, pourrait s'expliquer par l'incapacité des responsables à réparer monétairement les dommages pour différentes raisons : difficulté à repérer le dommage, à l'évaluer, à trouver le responsable, à reconstituer la chaîne de causalité entre le fait générateur et le dommage, à faire payer le responsable, ou encore à individualiser la compensation.

Enfin, il semble que les décideurs publics appliquent le plus souvent le principe de la compensabilité plutôt qu'un principe de compensation (Kolm, 1995). Ce principe prévoit qu'un projet doit être réalisé si les gagnants peuvent compenser les perdants sans exiger toutefois concrètement de flux

Tableau 4. Résultat attendu des requérants au tribunal administratif (TA)

\begin{tabular}{l|r|c}
\hline Attentes des requérants TA & Nb. cit. & Fréq. \\
\hline Abandon de l'usage ou du projet d'usage des mis en cause & 174 & $95 \%$ \\
\hline Compensation financière & 4 & $2 \%$ \\
\hline Demande d'expertise & 3 & $2 \%$ \\
\hline Aménagement de l'usage ou du projet d'usage des mis en cause & 2 & $1 \%$ \\
\hline TOTAL OBS. & 183 & $100 \%$ \\
\hline
\end{tabular}

Source : dépouillement par les auteurs des jugements du TA du Puy-de-Dôme (période 1998-2002) 
monétaires (qui de fait ne sont qu'hypothétiques). Devant cette situation, les opposants vont agir pour contourner les limites de la non-application de la compensation. Certes, ils revendiquent l'abandon des activités à l'origine des préjudices réels ou hypothétiques, mais surtout ils vont tenter, pour satisfaire leur demande, de capturer les instruments réglementaires d'organisation et de sélection des usages. En cas de réussite, l'usage privilégié à l'origine du préjudice est entravé et le bien-être des résidants est préservé car un projet ou une activité sont empêchés. L'action de contestation permet in fine, soit d'empêcher la création de perdants, soit de transformer les perdants en gagnants. C'est cette stratégie que nous allons analyser.

\section{La contestation des décisions publiques au tribunal administratif : obtenir leur annulation, empêcher l'usage}

Différents leviers d'action permettent aux opposants, d'une part d'exposer leurs préférences, et d'autre part de saisir les instruments de la réglementation administrative nécessaires pour contrôler l'usage incriminé

La presse quotidienne régionale est un observatoire intéressant des stratégies des parties en conflit. Les opposants exploitent diverses pistes. Ils exercent d'abord des pressions sur les responsables directs et indirects des externalités. Ils convoquent les supports médiatiques locaux en cherchant à élargir la base intéressée par le conflit afin de donner plus de force à leur opposition. Ils pressent la classe politique locale, voire même le préfet de rallier leur cause et d'agir en leur faveur. En outre, le quotidien La Montagne rapporte régulièrement des cas pour lesquels les opposants ont engagé un recours en justice, correspondant en fait à une requête devant le seul tribunal administratif.

Cet espace juridictionnel donne accès à la contestation des instruments réglementaires. Le contrôle de certains droits d'usage sur des biens tangibles permet indirectement de contrôler les droits d'usage des attributs des ressources communes convoitées. Pour jouir d'un cadre de vie sans les nuisances sonores des camions transportant les matériaux d'extraction d'une gravière voisine, les opposants ne chercheront pas à faire valoir un droit sur la quiétude de leur quartier, mais agiront pour faire modifier le code de la route local, ou empêcher l'attribution de l'autorisation d'exploiter la carrière, installation classée pour la protection de l'environnement (ICPE). S'ils obtiennent satisfaction les requérants acquièrent l'équivalent d'un droit à préserver leur capital spatial habitat. La gamme des instruments de droit que les opposants cherchent à entraver est large. Les résidants contestent majoritairement le contenu des arrêtés municipaux et préfectoraux. Il s'agit ici du contentieux pour excès de pouvoir, qui concerne des recours contre des actes administratifs. Les instruments réglementaires contestés régissent principalement des droits d'usage foncier et immobilier (zonage, permis de construire, occupation illégale, servitude de passage ou d'écoulement des eaux, remembrement, autorisation d'exploiter une ICPE).

Les arguments des opposants sont par ailleurs très divers dans certains différends. La difficulté principale des opposants est de trouver les moyens d'empêcher une activité qui est en général présumée légale. Aussi, les requérants, pour faire annuler une décision publique, cherchent souvent une faille sur la forme de la décision administrative : l'arrêté municipal ou préfectoral contiendra un vice de forme, la publication de l'arrêté n'aura pas été respectée, les délais de publications dépassés, rendant caduque la décision.

Les résultats des requérants au tribunal administratif sont majoritairement défavorables pour les défenseurs du cadre de vie qui agissent, rappelons-le, plus souvent à titre individuel. Seulement un tiers des requêtes rencontre un avis favorable du juge administratif, tout en ne concernant qu'exceptionnellement une réponse en terme de 
compensation monétaire (moins de $1 \%$ des cas). En revanche, les résultats obtenus dans les affaires relevées dans la presse quotidienne sont globalement positifs pour les opposants. Sur les 35 conflits portés par des défenseurs du cadre de vie résidentiel et impliquant les pouvoirs publics, les trois quart permettent d'obtenir la disparition ou la réduction du dommage. La compensation monétaire des dommages est un mode de résolution qui apparaît très marginale (un cas seulement). Cette situation d'abandon de projet ou d'usage a un effet direct sur la distribution des activités dans l'espace, sans que nous puissions en mesurer l'ampleur en termes de spécialisation des espaces. Toutefois, les abandons d'activités productives comme la fermeture de porcheries ou de carrières sous la pression d'usagers du cadre de vie résidentiel nous laissent bien supposer des réagencements des activités dans les campagnes.

\section{Conclusion}

Notre recherche montre l'intérêt d'analyser les caractéristiques des conflits d'usage du cadre de vie résidentiel pour mieux cerner les mutations contemporaines des espaces périurbains et ruraux notamment sur deux plans : l'intérêt porté au capital spatial habitat par les résidants et la remise en cause des choix publics locaux.

Tout d'abord, certains conflits d'usage en signalant d'abord l'incompatibilité entre les usages des attributs des ressources communes localisées de la campagne sont de bons indicateurs de la montée en puissance de la figure de la campagne cadre de vie. Ce travail met en évidence que les droits d'usage attribués par la puissance publique locale dans un contexte où un usage était préféré ne sont plus adaptés. Les normes sont comme mises en défaut par des consommateurs du cadre de vie résidentiel, qui ne sont plus en mesure d'accepter les contrats implicites qui contenaient les règles d'usage établies. Les usagers récepteurs des effets externes négatifs qui défendent leur capital spatial habitat (la qualité de l'air, le calme, le cadre paysager) revendiquent des droits d'usage sans toujours disposer de droit de propriété sur ces biens, ou de droit en responsabilité civile, mais font valoir leurs préférences pour ces biens en activant leur droit d'action notamment au tribunal administratif.

Ensuite, les conflits d'usage du cadre de vie résidentiel que nous avons analysés montrent qu'ils peuvent être aussi des leviers d'action d'un processus de marchandage par l'action individuelle ou collective entre des usagers du cadre de vie et des autorités chargées de mettre en œuvre les règles d'usages et/ou de fournir des biens publics locaux. Il ressort en effet que même dans les situations pour lesquelles les autorités publiques ne sont pas responsables directement du dommage matériel dont l'origine est le fait d'un agent privé, ce sont sur elles que porte prioritairement la contestation. Mais parce que la mise en œuvre de la compensation monétaire est difficile, ou parce qu'ils en refusent le principe, les opposants privilégient la stratégie d'empêchement de création des perdants, parfois en vain. Au vu de nos observations, il est possible que l'action médiatique et collective soit une voie qui permet d'obtenir plus souvent une amélioration du cadre de vie résidentiel que le recours individuel en contentieux administratif.

Il convient toutefois de rester prudent dans les interprétations plus générales que l'on peut tirer de ce travail : d'une part, nos résultats sont liés à deux sources distinctes qui ont conduit à un mode de constitution de nos observations différent, dont nous ne pouvons pas apprécier le biais ; d'autre part, ils restent partiels parce que liés à ces deux seules sources. Signalons toutefois qu'une analyse du contentieux judiciaire (civil et pénal) que nous avons réalisée depuis (étude à paraître) montre que la compensation monétaire des dommages et 
intérêts au civil comme la réparation monétaire civile au pénal concernent seulement environ un quart des affaires.

Les deux catégories des conflits concernant la contestation des décisions publiques locales sur lesquelles nous avons centré l'analyse sont des leviers possibles d'ajustement plus fins des choix publics aux préférences des résidants. Il s'ensuit dans de nombreuses situations une nouvelle distribution des droits d'usage censée améliorer le produit social net. Le conflit apparaît alors en quelque sorte comme un mode complémentaire à l'action du décideur public local en charge d'allouer des ressources communes. Mais le conflit en contribuant également à une nouvelle distribution spatiale des usages joue un rôle sans doute non négligeable sur la physionomie des espaces périurbains et ruraux en termes de spécialisation ou au contraire de multifonctionnalité des campagnes. Ce dernier point mériterait, de notre point de vue, d'être exploré.

\section{RÉFÉRENCES BIBLIOGRAPHIQUES}

Baumol W.-J., Oates W.-E. (1988). The theory of environmental policy. New York, Cambridge University Press, 299 p.

Buchanan J.-M., Tullock G. (1962). The calculus of consent, Logical foundations of constitutional Democracy. University of Michigan Press - Ann Arbor paperbacks, $361 \mathrm{p}$.

Caron A., Rialland C. (2001). Recension des travaux portant sur les conflits d'usages dans l'espace rural et propositions d'éléments pour une typologie. Paris, DATAR, Groupe de prospective «Espaces naturels et ruraux et société urbanisée », $38 \mathrm{p}$.

Catin M. (1985). Effets externes. Marché et systèmes de décision collective. Paris, Cujas, 455 p.

Charlier B. (1999). La défense de l'environnement : entre espace et territoire, géographie des conflits environnementaux déclenchés en France depuis 1974. Thèse de doctorat en géographie, Université de Pau et des pays de l'Adour, département de géographie, $753 \mathrm{p}$.

Daubresse M. (2003). La reprise de l'accession à la propriété. INSEE Première, $\mathrm{n}^{\circ}$ 913, juillet 2003, p. 4.

Deutsch M. (1994). Constructive conflict resolution: principles, training, and research. Journal of social issues, vol. $50, \mathrm{n}^{\circ} 1$, p. 13-32.
Guérin M. (2005). Conflits d'usage à l'horizon 2020 - Quels nouveaux rôles pour l'État dans les espaces ruraux et périurbains ? Paris, Commissariat Général du Plan, $200 \mathrm{p}$.

Guillain P.-E. (2001). Les conflits d'usage dans les campagnes à travers la presse quotidienne régionale. In INRA CESAER, Dijon, Rapport de stage deuxième année INA-PG, sous la direction de Philippe Perrier-Cornet, p. 19 + annexes.

Hirschman A.-O. (1970). Exit, Voice and Loyalty. Harvard, Harvard University Press, 144 p.

Hussenet E. (Dir.) (2002). Résistez - Les vrais raisons de lutter, les outils pour se faire entendre. Bayeux, Village éditions, $318 \mathrm{p}$.

Jackman R.-A. (1975). The Problem of Externalities in a Spatial Economy. In CRIPPS E.-L. (Dir.), Regional Science New Concepts and Old Problems, Pion Publication, p. 18-30.

Jeanneaux P. (2006). Les conflits d'usage dans les espaces périurbain et ruraux français - une approche par l'analyse économique de la décision publique. Doctorat en sciences économiques, Dijon, Université de Bourgogne, UFR de sciences économiques, 252 p. + annexes. 
Jessua C. (1991). Histoire de la théorie économique. Paris, PUF, Économie, 584 p.

Kolm S.-C. (1995). Sens ou Non-sens du calcul économique public : le principe de compensation. Entreprise Éthique, vol. 2, avril, p. 85-93.

Lévy J. (1994). L'espace légitime - Sur la dimension géographique de la fonction politique. Paris, Presses de la Fondation Nationale des Sciences Politiques, $442 \mathrm{p}$.

Libecap G.-D. (1989). Contracting for property rights. Cambridge, Cambridge University Press, $132 \mathrm{p}$.

Mishan E.-J. (1969). The Relationship between Joint Products, Collective Goods and External Effects, Journal of Political Economy, vol. LXXVII, May June, p. 342-345.

Passet R. (1979). L'économique et le vivant. Paris, Payot, 287 p.

Perrier-Cornet P. (2002). Repenser les campagnes. Éditions de l'Aube-Datar, La Tour d'Aigues, 279 p.

Plateau C., Rakotomalaia J. (2005). Construction neuve - L'attrait des maisons individuelles en milieu rural mais proches des villes. SESP en Bref, $\mathrm{n}^{\circ} 1$, juillet, p. 4.

Point P. (1997). La valeur de l'eau comme actif naturel multifonction. Compte rendu de l'Académie d'Agriculture de France, vol. 83, n 4, p. 179-194.
Quirion P. (1999). Le marché de l'assurance $d u$ risque pollution en France. Paris, CERNA - Écoles des Mines de Paris, Étude pour le ministère de l'Aménagement du Territoire et de l'Environnement (DGAD-SRAE), sous la direction de Pierre-Noël Giraud, 59 p.

Richman B.-D., Boerner C. (2004). A Transaction Cost Economizing Approach to Regulation: Understanding the NIMBY Problem and Improving Regulatory Responses. American Law and Economics Association - Fourteenth Annual Meeting, Chicago, Northwestern University School of Law, May 7-8, 71 p.

Ruff L.-E. (1970). The economic sense of pollution. The Public Interest, vol. 19, p. 69-85.

Simmel G. (1995). Le conflit. Dijon, Circé, $159 \mathrm{p}$.

Torre A., Aznar O., Bonin M., Caron A., Chia E., Galman M., Guerin M., Jeanneaux P., Kirat T., Lefranc C., Paoli J.-C., Salazar M.-I., Thinon P. (2006). Conflits et tensions autour des usages de l'espace dans les territoires ruraux et péri-urbains. Le cas de six zones géographiques françaises. Revue d'économie régionale et urbaine, $\mathrm{n}^{\circ} 3$, p. 415-453.

Vigouroux I. (1999). Asymétries informationnelles et marché politique. Paris IPanthéon Sorbonne, UFR d'économie, Sciences économiques, $311 \mathrm{p}$. 